\title{
ASYMPTOTIC BOUNDARY OBSERVABILITY FOR THE WAVE EQUATION ON ONE SIDE OF A PLANAR TRIANGLE
}

\author{
HANS CHRISTIANSON AND EVAN STAFFORD
}

\begin{abstract}
We consider the wave equation $\left(\partial_{t}^{2}-\Delta\right) u=0$ on a planar triangular domain $\Omega \subset \mathbb{R}^{2}$ with Dirichlet boundary conditions. We use a commutator and integration by parts argument similar to that in Chr17 by the first author to obtain an observability asymptotic for any one side of the triangle. Our result is particular to triangular domains and does not hold for polygons in general.
\end{abstract}

\section{INTRODUCTION}

In this paper, we study boundary observability for solutions to the wave equation on flat triangles. The main result is a large time asymptotic observability identity from any one side. In the closely related work Chr17 by the first author, it is shown that the Neumann data for Dirichlet eigenfunctions on a triangle is equidistributed on each side. Moreover, it is an exact equality, so it is reasonable to expect a similar statement for solutions to the wave equation. We formulate this statement in terms of an observability identity which roughly asks "can we detect a wave by making a measurement at the boundary?"

The traditional methods for understanding control and observability use geometric optics and microlocal analysis, however our approach does not. Instead, our result only uses a robust commutator and integration by parts argument. We begin the proof by computing the commutator of the wave operator and an appropriate radial vector field. By using this computation in tandem with evaluating the commutator explicitly, we obtain an equation from which we derive our result, after applying a few rudimentary estimates. The paper is entirely self-contained.

Let $\Omega$ be a triangular region in $\mathbb{R}^{2}$, and suppose the sides are labeled $A, B$, and $C$. Let $\ell_{A}$ (respectively $\ell_{B}, \ell_{C}$ ) denote the altitude corresponding to $A$ (respectively $B, C)$, meaning the perpendicular distance from the line containing $A, B$, or $C$ to the non-adjacent corner (see Figures 1 and 2 for a picture). Let $L$ denote the length of the longest side. Our main theorem is the following:

Theorem 1. Consider the following initial/boundary value problem for the wave equation:

$$
\left\{\begin{array}{l}
\left(\partial_{t}^{2}-\Delta\right) u=0 \\
\left.u\right|_{\partial \Omega}=0 \\
u(0, x, y)=u_{0}(x, y), u_{t}(0, x, y)=u_{1}(x, y)
\end{array}\right.
$$


where $u_{0}, u_{1} \in H_{0}^{1}(\Omega) \cap H^{s}(\Omega)$ for all $s \geq 0$. Then for all $T>0$, the Neumann data on side $A$ satisfies

$$
\int_{0}^{T} \int_{A}\left|\partial_{\nu} u\right|^{2} d S d t=\frac{T}{\ell_{A}} E(0)\left(1+\mathcal{O}\left(\frac{L}{T}\right)\right)
$$

where $\partial_{\nu}$ is the normal derivative on $\partial \Omega, d S$ is the arclength measure, and $E(0)$ is the (conserved) initial energy, defined by

$$
E(t)=\int_{\Omega}\left|\partial_{t} u\right|^{2}+|\nabla u|^{2} d V
$$

The analogous asymptotic on sides $B$ and $C$ also holds.

Remark 1 . This is not a low-regularity result; the assumptions on the regularity of $u_{0}$ and $u_{1}$ are simply to allow us to integrate by parts as necessary.

Remark 2. Note that if $\partial_{\nu} u=0$ on any one of the sides, we have that $E(0)=0$, which implies $u=0$. In particular, given two solutions $u$ and $v$ to 1.1 with identical Neumann data on at least one side, we have in fact that $u-v=0$ everywhere. Thus, Theorem 1 can be seen to imply a uniqueness condition from Neumann data on just one side of the triangle.

Remark 3. It is important that the domain $\Omega$ is a triangle, as this theorem fails in general if $\Omega$ is merely assumed to be polygonal. This is demonstrated in Section 4 . where we show that the result fails for square domains.

A similar result is believed to hold in higher dimensions and we will return to this question later.

Remark 4. The appearance of the factor $1 / \ell_{A}$ in 1.2 can be seen as a consequence of finite propagation speed, since it takes time $\sim \ell_{A}$ for a wave to travel from the opposite corner to side $A$.

Remark 5 (History). For control and observability for solutions to the wave equation, a landmark result is one of Rauch-Taylor RT74], who introduced the use of rays from geometric optics into the study of interior control problems. For boundary contol and observability as studied in this paper, an intuitive condition for the region of control to satisfy in order to establish observability is that every ray hits the region in some finite time. This condition was shown to be necessary by a result of Ralston [Ral69], and RT74] showed that for manifolds without boundary and interior control, this condition is (roughly) sufficient as well. Following this is an important result of Bardos-Lebeau-Rauch BLR92, which showed sufficiency for the case of observation and control problems on bounded domains for which the region of control lies on the boundary.

There are also results for cases where this geometric control condition is not fully satisfied. Lebeau Leb96 considered a particular example of a damped wave problem on a manifold where the damping region does not meet every geometric ray, but has enough control that the energy still decays exponentially with some derivative loss. The papers Chr07 along with Chr10 by the first author built on this example and obtained a sub-exponential estimate that holds for a wider class of operators and manifolds. The paper BC15 demonstrated that this estimate is in general sharp, but can be improved with the addition of a stronger damping term. 


\section{Simplified Cases}

2.1. Case of a Single Dirichlet Eigenfunction. We first consider a case where we can make an explicit calculation. Let $\varphi(x, y)$ be a Dirichlet eigenfunction on a triangle $\Omega$ satisfying

$$
\left\{\begin{array}{l}
\left(-\Delta-\lambda^{2}\right) \varphi=0 \\
\left.\varphi\right|_{\partial \Omega}=0
\end{array}\right.
$$

normalized so that $\|\varphi\|_{L^{2}(\Omega)}=1$. Note that by the main result of Chr17, we have that

$$
\int_{A}\left|\partial_{\nu} \varphi\right|^{2} d S=\frac{\lambda^{2} a}{\operatorname{Area}(\Omega)}=\frac{\lambda^{2} a}{a \ell_{A} / 2}=\frac{2 \lambda^{2}}{\ell_{A}}
$$

where $a$ is the length of side $A$. Now define

$$
u(t, x, y)=\sin (t \lambda) \varphi(x, y)
$$

so $u$ satisfies

$$
\left\{\begin{array}{l}
\left(\partial_{t}^{2}-\Delta\right) u=0 \\
\left.u\right|_{\partial \Omega}=0 \\
u(0, x, y)=0, \partial_{t} u(0, x, y)=\lambda \varphi(x, y)
\end{array}\right.
$$

Note that for solutions to the wave equation 1.1 , the energy 1.3 is conserved:

$$
\begin{aligned}
\frac{d}{d t} E(t)= & \int_{\Omega} \partial_{t} \bar{u} \partial_{t}^{2} u+\partial_{t} u \partial_{t}^{2} \bar{u}+\left(\partial_{t} \nabla \bar{u}\right) \cdot(\nabla u)+\left(\partial_{t} \nabla u\right) \cdot(\nabla \bar{u}) d V \\
= & \int_{\Omega} \partial_{t} \bar{u} \partial_{t}^{2} u+\partial_{t} u \partial_{t}^{2} \bar{u}-\partial_{t} \bar{u} \Delta u-\partial_{t} u \Delta \bar{u} d V \\
& \quad+\int_{\partial \Omega} \partial_{t} \bar{u} \partial_{\nu} u+\partial_{t} u \partial_{\nu} \bar{u} d S \\
= & \int_{\Omega} \partial_{t} \bar{u}\left(\partial_{t}^{2} u-\Delta u\right)+\partial_{t} u\left(\partial_{t}^{2} \bar{u}-\Delta \bar{u}\right) d V \\
= & 0
\end{aligned}
$$

by integration by parts and using the conditions in 1.1. In this case, since $\|\varphi\|_{L^{2}(\Omega)}=1$, the energy is

$$
\begin{aligned}
E(0) & =\int_{\Omega}\left|\partial_{t} u(0, x, y)\right|^{2}+|\nabla u(0, x, y)|^{2} d V \\
& =\int_{\Omega} \lambda^{2}|\varphi|^{2} d V \\
& =\lambda^{2} .
\end{aligned}
$$


We can then compute explicitly

$$
\begin{aligned}
\int_{0}^{T} \int_{A}\left|\partial_{\nu} u\right|^{2} d S d t & =\int_{0}^{T} \int_{A} \sin ^{2}(t \lambda)\left|\partial_{\nu} \varphi\right|^{2} d S d t \\
& =\int_{0}^{T} \frac{1}{2}(1-\cos (2 t \lambda)) \int_{A}\left|\partial_{\nu} \varphi\right|^{2} d S d t \\
& =\frac{\lambda^{2}}{\ell_{A}}\left(T-\frac{1}{2 \lambda} \sin (2 T \lambda)\right) \\
& =\frac{T}{\ell_{A}} E(0)\left(1-\frac{1}{2 T \lambda} \sin (2 T \lambda)\right)
\end{aligned}
$$

and we see that the result is realized in this case.

As Dirichlet eigenfunctions $\left\{\varphi_{k}\right\}_{k=1}^{\infty}$ form an orthonormal basis of $L^{2}(\Omega)$, we may ask whether our main result could be established by a similar calculation after noting that a solution $u$ to 1.1 may be expressed as an expansion of the form

$$
u(t, x, y)=\sum_{k=1}^{\infty}\left(a_{k} e^{i t \lambda_{k}}+b_{k} e^{-i t \lambda_{k}}\right) \varphi_{k}(x, y)
$$

Unfortunately, at this time this is not possible. In the computation of $\int_{A}\left|\partial_{\nu} u\right|^{2} d S$ we would in general obtain cross terms, and we do not have that the $\left.\partial_{\nu} \varphi_{k}\right|_{\partial \Omega}$ are orthogonal in $L^{2}(\partial \Omega)$. Thus, another approach is needed, which we will first demonstrate for the simpler one-dimensional case in $\$ 2.2$ and later adapt to the two-dimensional case for the main proof in Section 3 .

2.2. One-Dimensional Problem. Here we present the one-dimensional analog of the problem stated in Theorem 1 in order to establish our general approach.

Theorem 2. Let $\Omega \subset \mathbb{R}$ be the interval $[0, \ell]$, and consider the following initial/boundary value problem for the wave equation:

$$
\left\{\begin{array}{l}
\left(\partial_{t}^{2}-\partial_{x}^{2}\right) u=0 \\
u(t, 0)=u(t, \ell)=0 \\
u(0, x)=u_{0}(x), u_{t}(0, x)=u_{1}(x)
\end{array}\right.
$$

where $u_{0}, u_{1} \in H_{0}^{1}(\Omega) \cap H^{s}(\Omega)$ for all $s \geq 0$. Then for all $T>0$, the Neumann data at the endpoint $x=\ell$ satisfies

$$
\left.\int_{0}^{T}\left|\partial_{x} u\right|^{2}\right|_{x=\ell} d t=\frac{T}{\ell} E(0)\left(1+\mathcal{O}\left(\frac{\ell}{T}\right)\right)
$$

where $E(0)$ is the (conserved) initial energy, defined by

$$
E(t)=\int_{0}^{\ell}\left|\partial_{t} u\right|^{2}+\left|\partial_{x} u\right|^{2} d x .
$$


To see that the energy is conserved, we compute

$$
\begin{aligned}
\frac{d}{d t} E(t) & =\frac{d}{d t} \int_{0}^{\ell}\left|\partial_{t} u\right|^{2}+\left|\partial_{x} u\right|^{2} d x \\
& =\int_{0}^{\ell} \partial_{t}^{2} u \partial_{t} \bar{u}+\partial_{t} u \partial_{t}^{2} \bar{u}+\partial_{x} \partial_{t} u \partial_{x} \bar{u}+\partial_{x} u \partial_{x} \partial_{t} \bar{u} d x \\
& =\int_{0}^{\ell} \partial_{t}^{2} u \partial_{t} \bar{u}+\partial_{t} u \partial_{t}^{2} \bar{u}-\partial_{t} u \partial_{x}^{2} \bar{u}-\partial_{x}^{2} u \partial_{t} \bar{u} d x+\left.\left[\partial_{t} u \partial_{x} \bar{u}+\partial_{x} u \partial_{t} \bar{u}\right]\right|_{0} ^{\ell} \\
& =\int_{0}^{\ell}\left(\partial_{t}^{2} u-\partial_{x}^{2} u\right) \partial_{t} \bar{u}+\left(\partial_{t}^{2} \bar{u}-\partial_{x}^{2} \bar{u}\right) \partial_{t} u d x \\
& =0
\end{aligned}
$$

by integration by parts and using the conditions in $(2.9)$.

Consider the vector field

$$
X=x \partial_{x}
$$

on $\Omega$. We have the commutator

$$
\begin{aligned}
{\left[\partial_{t}^{2}-\partial_{x}^{2}, X\right] } & =\left(\partial_{t}^{2}-\partial_{x}^{2}\right) x \partial_{x}-x \partial_{x}\left(\partial_{t}^{2}-\partial_{x}^{2}\right) \\
& =-\partial_{x}^{2} x \partial_{x}+x \partial_{x}^{3} \\
& =-\partial_{x}\left(\partial_{x}+x \partial_{x}^{2}\right)+x \partial_{x}^{3} \\
& =-\partial_{x}^{2}-\partial_{x}^{2}-x \partial_{x}^{3}+x \partial_{x}^{3} \\
& =-2 \partial_{x}^{2} .
\end{aligned}
$$

Using this, along with integration by parts and the fact that $u$ satisfies the wave equation, we obtain

$$
\begin{aligned}
\int_{0}^{T} \int_{0}^{\ell}\left[\partial_{t}^{2}-\partial_{x}^{2}, X\right] u \bar{u} d x d t= & \int_{0}^{T} \int_{0}^{\ell}-2 \partial_{x}^{2} u \bar{u} d x d t \\
= & \int_{0}^{T} \int_{0}^{\ell}\left(-\partial_{t}^{2}-\partial_{x}^{2}\right) u \bar{u} d x d t \\
= & \int_{0}^{T} \int_{0}^{\ell}\left|\partial_{t} u\right|^{2}+\left|\partial_{x} u\right|^{2} d x d t \\
& \quad-\left.\int_{0}^{\ell} \partial_{t} u \bar{u} d x\right|_{0} ^{T}-\left.\int_{0}^{T} \partial_{x} u \bar{u}\right|_{0} ^{\ell} d t
\end{aligned}
$$

We recognize the energy in the first term, and we note that the third term vanishes by the boundary conditions in 2.9 , , so

$$
\begin{aligned}
\int_{0}^{T} \int_{0}^{\ell}\left[\partial_{t}^{2}-\partial_{x}^{2}, X\right] u \bar{u} d x d t & =\int_{0}^{T} E(0) d t-\left.\int_{0}^{\ell} \partial_{t} u \bar{u} d x\right|_{0} ^{T} \\
& =T E(0)-\left.\int_{0}^{\ell} \partial_{t} u \bar{u} d x\right|_{0} ^{T} .
\end{aligned}
$$


From the starting point of 2.15 , we now evaluate the commutator explicitly, which yields

$$
\begin{aligned}
\int_{0}^{T} \int_{0}^{\ell}\left[\partial_{t}^{2}-\partial_{x}^{2}, X\right] u \bar{u} d x d t= & \int_{0}^{T} \int_{0}^{\ell}\left(\partial_{t}^{2}-\partial_{x}^{2}\right) x \partial_{x} u \bar{u}-x \partial_{x}\left(\partial_{t}^{2}-\partial_{x}^{2}\right) u \bar{u} d x d t \\
= & \int_{0}^{T} \int_{0}^{\ell} \partial_{t}^{2} x \partial_{x} u \bar{u}-\partial_{x}^{2} x \partial_{x} u \bar{u} d x d t \\
= & \int_{0}^{T} \int_{0}^{\ell}-\left(\partial_{t}\left(x \partial_{x} u\right)\right)\left(\partial_{t} u\right)+\left(\partial_{x}\left(x \partial_{x} u\right)\right)\left(\partial_{x} \bar{u}\right) d x d t \\
& +\left.\int_{0}^{\ell}\left(\partial_{t}\left(x \partial_{x} u\right)\right) \bar{u} d x\right|_{0} ^{T}-\left.\int_{0}^{T}\left(\partial_{x}\left(x \partial_{x} u\right)\right) \bar{u}\right|_{0} ^{\ell} d t
\end{aligned}
$$

again by integration by parts and the fact that $u$ satisfies the wave equation. Note that the third term vanishes by the boundary conditions. We may integrate by parts in the first term again and use the fact that $\bar{u}$ also satisfies the wave equation to obtain

$$
\begin{aligned}
\int_{0}^{T} \int_{0}^{\ell}\left[\partial_{t}^{2}-\partial_{x}^{2}, X\right] u \bar{u} d x d t= & \int_{0}^{T} \int_{0}^{\ell}\left(x \partial_{x} u\right)\left(\partial_{t}^{2} \bar{u}\right)-\left(x \partial_{x} u\right)\left(\partial_{x}^{2} \bar{u}\right) d x d t \\
& +\int_{0}^{\ell}\left(\partial_{t}\left(x \partial_{x} u\right)\right) \bar{u}-\left.\left(x \partial_{x} u\right)\left(\partial_{t} \bar{u}\right) d x\right|_{0} ^{T} \\
& +\left.\int_{0}^{T}\left(x \partial_{x} u\right)\left(\partial_{x} \bar{u}\right)\right|_{0} ^{\ell} d t \\
= & \int_{0}^{\ell}\left(\partial_{t}\left(x \partial_{x} u\right)\right) \bar{u}-\left.\left(x \partial_{x} u\right)\left(\partial_{t} \bar{u}\right) d x\right|_{0} ^{T} \\
& +\left.\int_{0}^{T} \ell\left|\partial_{x} u\right|^{2}\right|_{x=\ell} d t
\end{aligned}
$$

Using the fact that $x \partial_{x} u=\partial_{x}(x u)-u$, we can then integrate by parts yet again to arrive at

$$
\begin{aligned}
\int_{0}^{T} \int_{0}^{\ell}\left[\partial_{t}^{2}-\partial_{x}^{2}, X\right] u \bar{u} d x d t= & \int_{0}^{\ell}\left(\partial_{t}\left(\partial_{x}(x u)\right) \bar{u}-\partial_{t} u \bar{u}-\left.\left(x \partial_{x} u\right)\left(\partial_{t} \bar{u}\right) d x\right|_{0} ^{T}\right. \\
& +\left.\int_{0}^{T} \ell\left|\partial_{x} u\right|^{2}\right|_{x=\ell} d t \\
= & \int_{0}^{\ell}-\left(\partial_{t} u\right)\left(x \partial_{x} \bar{u}\right)-\partial_{t} u \bar{u}-\left.\left(x \partial_{x} u\right)\left(\partial_{t} \bar{u}\right) d x\right|_{0} ^{T} \\
& +\left.\int_{0}^{T} \ell\left|\partial_{x} u\right|^{2}\right|_{x=\ell} d t+\left.\left.\partial_{t}(x u) \bar{u}\right|_{x=0} ^{\ell}\right|_{t=0} ^{T} \\
= & \int_{0}^{\ell}-\left(\partial_{t} u\right)\left(x \partial_{x} \bar{u}\right)-\partial_{t} u \bar{u}-\left.\left(x \partial_{x} u\right)\left(\partial_{t} \bar{u}\right) d x\right|_{0} ^{T} \\
& +\left.\int_{0}^{T} \ell\left|\partial_{x} u\right|^{2}\right|_{x=\ell} d t .
\end{aligned}
$$


Combining this with 2.16) and reorganizing terms then yields

$$
\left.\int_{0}^{T} \ell\left|\partial_{x} u\right|^{2}\right|_{x=\ell} d t=T E(0)+\int_{0}^{\ell}\left(\partial_{t} u\right)\left(x \partial_{x} \bar{u}\right)+\left.\left(x \partial_{x} u\right)\left(\partial_{t} \bar{u}\right) d x\right|_{0} ^{T} .
$$

Note that in this case, the $\left.\int_{0}^{\ell} \partial_{t} u \bar{u} d x\right|_{0} ^{T}$ terms from 2.16) and 2.19) cancel. This does not occur in the two-dimensional case, where these extra terms will instead require us to make additional estimates to control them.

We now obtain bounds for the integral on the right hand side of 2.20. By Cauchy's inequality with parameter $\alpha>0$ we have

$$
\begin{aligned}
\int_{0}^{\ell}\left|\left(\partial_{t} u\right)\left(x \partial_{x} \bar{u}\right)\right| d x & \leq \int_{0}^{\ell} \frac{\alpha}{2}\left|\partial_{t} u\right|^{2}+\frac{1}{2 \alpha}\left|x \partial_{x} u\right|^{2} d x \\
& \leq \int_{0}^{\ell} \frac{\alpha}{2}\left|\partial_{t} u\right|^{2}+\frac{\ell^{2}}{2 \alpha}\left|\partial_{x} u\right|^{2} d x
\end{aligned}
$$

Taking $\alpha=\ell$, this becomes

$$
\int_{0}^{\ell}\left|\left(\partial_{t} u\right)\left(x \partial_{x} \bar{u}\right)\right| d x \leq \frac{\ell}{2} E(0) .
$$

Equation 2.20 then implies simultaneously

$$
\left.\int_{0}^{T} \ell\left|\partial_{x} u\right|^{2}\right|_{x=\ell} d t \leq T E(0)+4 \frac{\ell}{2} E(0)
$$

and

$$
\left.\int_{0}^{T} \ell\left|\partial_{x} u\right|^{2}\right|_{x=\ell} d t \geq T E(0)-4 \frac{\ell}{2} E(0)
$$

which yield the asymptotic

$$
\left.\int_{0}^{T}\left|\partial_{x} u\right|^{2}\right|_{x=\ell} d t=\frac{T}{\ell} E(0)\left(1+\mathcal{O}\left(\frac{\ell}{T}\right)\right) .
$$

This is the one-dimensional analog of the main result. We now move on to the proof of the two dimensional case.

\section{Proof of the Main Theorem}

3.1. Proof for Acute Triangles. The proof of the main theorem is split into two cases, one for acute triangles and one for obtuse (or right) triangles. The steps in both cases are the same in spirit, and in fact the proof from the acute case works for all but one side in the obtuse case. Without loss in generality, we prove the main theorem for side $A$ only, and use $\ell=\ell_{A}$ to ease notation.

We use a setup similar to that in [Chr17]. Suppose $\Omega$ is an acute triangle, as in Figure 1. Let $\Omega$ be oriented such that the vertex opposing $A$ is located at the origin, and $A$ is perpendicular to the $x$-axis. Note that this causes the altitude of length $\ell$ to coincide with the $x$-axis. Label the remaining sides $B$ and $C$ in a clockwise fashion. Let $a_{1}$ be the length of the part of $A$ below the $x$-axis, and similarly let $a_{2}$ be the length of the part above. Consider the problem (1.1) and define the vector field

$$
X=x \partial_{x}+y \partial_{y}
$$




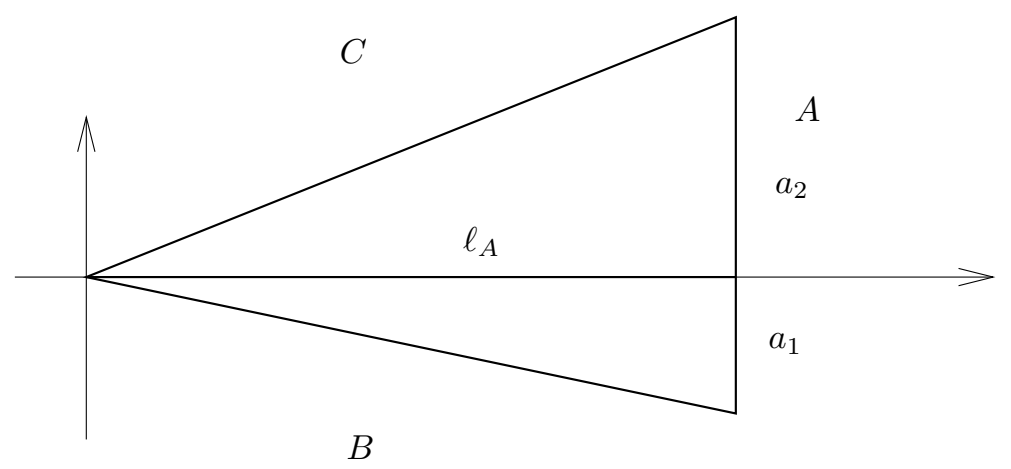

Figure 1. Setup for acute triangles.

on $\Omega$. We have the commutator

$$
\begin{aligned}
{\left[\partial_{t}^{2}-\Delta, X\right] } & =\left(\partial_{t}^{2}-\Delta\right) X-X\left(\partial_{t}^{2}-\Delta\right) \\
& =-\Delta x \partial_{x}-\Delta y \partial_{y}+X \Delta \\
& =-2 \partial_{x}^{2}-x \partial_{x} \Delta-2 \partial_{y}^{2}-y \partial_{y} \Delta+X \Delta \\
& =-2 \Delta .
\end{aligned}
$$

From this and the fact that $u$ satisfies the wave equation, by integration by parts we have

$$
\begin{aligned}
\int_{0}^{T} \int_{\Omega}\left[\partial_{t}^{2}-\Delta, X\right] u \bar{u} d V d t & =\int_{0}^{T} \int_{\Omega}(-2 \Delta u) \bar{u} d V d t \\
& =\int_{0}^{T} \int_{\Omega}\left(-\Delta u-\partial_{t}^{2} u\right) \bar{u} d V d t \\
& =\int_{0}^{T} \int_{\Omega}|\nabla u|^{2}+\left|\partial_{t} u\right|^{2} d V d t+\int_{\Omega}-\left.\partial_{t} u \bar{u} d V\right|_{0} ^{T} \\
& =T E(0)+\int_{\Omega}-\left.\partial_{t} u \bar{u} d V\right|_{0} ^{T}
\end{aligned}
$$

mirroring what was obtained in the one-dimensional case. Also, by evaluating the commutator explicitly and integrating by parts, we obtain

$$
\begin{aligned}
\int_{0}^{T} \int_{\Omega}\left[\partial_{t}^{2}-\Delta, X\right] u \bar{u} d V d t= & \int_{0}^{T} \int_{\Omega}\left(\partial_{t}^{2}-\Delta\right) X u \bar{u}-X\left(\partial_{t}^{2}-\Delta\right) u \bar{u} d V d t \\
= & \int_{0}^{T} \int_{\Omega} \partial_{t}^{2} X u \bar{u}-\Delta X u \bar{u} d V d t \\
= & \int_{0}^{T} \int_{\Omega}-\left(\partial_{t} X u\right)\left(\partial_{t} \bar{u}\right)+(\nabla X u) \cdot(\nabla \bar{u}) d V d t \\
& \quad+\left.\int_{\Omega}\left(\partial_{t} X u\right) \bar{u} d V\right|_{0} ^{T}-\int_{0}^{T} \int_{\partial \Omega}\left(\partial_{\nu} X u\right) \bar{u} d S d t
\end{aligned}
$$


The third term vanishes since $\left.\bar{u}\right|_{\partial \Omega}=0$. Integrating by parts again in the first term then gives

$$
\begin{aligned}
\int_{0}^{T} \int_{\Omega}\left[\partial_{t}^{2}-\Delta, X\right] u \bar{u} d V d t= & \int_{0}^{T} \int_{\Omega}(X u)\left(\partial_{t}^{2} \bar{u}\right)-(X u)(\Delta \bar{u}) d V d t \\
& +\int_{\Omega}\left(\partial_{t} X u\right) \bar{u}-\left.(X u)\left(\partial_{t} \bar{u}\right) d V\right|_{0} ^{T} \\
& +\int_{0}^{T} \int_{\partial \Omega}(X u)\left(\partial_{\nu} \bar{u}\right) d S d t
\end{aligned}
$$

Since $\bar{u}$ also satisfies the wave equation, the first term on the right disappears. Using the fact that $X u=-2 u+\partial_{x}(x u)+\partial_{y}(y u)$ and integrating by parts in the second term (the boundary term vanishes by the boundary conditions) then yields

$$
\begin{aligned}
\int_{0}^{T} \int_{\Omega}\left[\partial_{t}^{2}-\Delta, X\right] u \bar{u} d V d t=\int_{\Omega} & -2 \partial_{t} u \bar{u}-\left(\partial_{t} u\right)(X \bar{u})-\left.(X u)\left(\partial_{t} \bar{u}\right) d V\right|_{0} ^{T} \\
& +\int_{0}^{T} \int_{\partial \Omega}(X u)\left(\partial_{\nu} \bar{u}\right) d S d t
\end{aligned}
$$

Note that this is slightly different from what was obtained in the one-dimensional case due to the factor of 2 in the first term on the right hand side. Combining this with $(3.3)$ and reorganizing terms gives

$$
\int_{0}^{T} \int_{\partial \Omega}(X u)\left(\partial_{\nu} \bar{u}\right) d S d t=T E(0)+\int_{\Omega} \partial_{t} u \bar{u}+\left(\partial_{t} u\right)(X \bar{u})+\left.(X u)\left(\partial_{t} \bar{u}\right) d V\right|_{0} ^{T}
$$

and we see that there is a new term on the right which we must also account for.

First, we can obtain bounds in terms of the energy $E(0)$ for the latter terms on the right hand side in a similar fashion to the one-dimensional case. Note that $|x|$ and $|y|$ are bounded by $L$ ( $L=$ length of longest side as in the introduction) on $\Omega$ due to our choice of orientation for the triangle. An application of Cauchy's inequality with parameter $\alpha>0$ and the triangle inequality then implies

$$
\begin{aligned}
\int_{\Omega}\left|\left(\partial_{t} u\right) X \bar{u}\right| d V & \leq \int_{\Omega} \frac{\alpha}{2}\left|\partial_{t} u\right|^{2}+\frac{1}{2 \alpha}|X u|^{2} d V \\
& \leq \int_{\Omega} \frac{\alpha}{2}\left|\partial_{t} u\right|^{2}+\frac{L^{2}}{2 \alpha}\left(\left|\partial_{x} u\right|+\left|\partial_{y} u\right|\right)^{2} d V \\
& =\int_{\Omega} \frac{\alpha}{2}\left|\partial_{t} u\right|^{2}+\frac{L^{2}}{2 \alpha}|\nabla u|^{2}+\frac{L^{2}}{\alpha}\left|\partial_{x} u\right|\left|\partial_{y} u\right| d V
\end{aligned}
$$

Applying Cauchy's inequality again then implies

$$
\begin{aligned}
\int_{\Omega}\left|\left(\partial_{t} u\right)(X \bar{u})\right| d V & \leq \int_{\Omega} \frac{\alpha}{2}\left|\partial_{t} u\right|^{2}+\frac{L^{2}}{2 \alpha}|\nabla u|^{2}+\frac{L^{2}}{2 \alpha}|\nabla u|^{2} d V \\
& \leq \frac{L}{\sqrt{2}} E(0)
\end{aligned}
$$

where $\alpha=L \sqrt{2}$ is chosen. 
For the new term, note that by Cauchy's inequality with parameter $\beta>0$,

$$
\begin{aligned}
\left|\int_{\Omega} \partial_{t} u \bar{u} d V\right| & \leq\left\|\partial_{t} u\right\|_{L^{2}(\Omega)}\|u\|_{L^{2}(\Omega)} \\
& \leq \frac{\beta}{2}\left\|\partial_{t} u\right\|_{L^{2}(\Omega)}^{2}+\frac{1}{2 \beta}\|u\|_{L^{2}(\Omega)}^{2} .
\end{aligned}
$$

We now establish a Poincaré-type inequality. We present a full proof here since it is very simple in the case of a triangle.

Lemma 1. Let $u \in H_{0}^{1}(\Omega) \cap H^{s}(\Omega)$ for every $s$. Then the following holds:

$$
\|u\|_{L^{2}(\Omega)} \leq L \sqrt{e-1}\left\|\partial_{x} u\right\|_{L^{2}(\Omega)} .
$$

We observe that the right hand side involves only the $x$ derivative of $u$. This allows us to bound the right hand side of 3.10 using the energy after an appropriate choice of $\beta$. Such inequalities have been widely studied as described in [KN15], which notes results such as Poincaré [Poi90 and Steklov Ste97]. We note that if we happen to know the first Dirichlet eigenvalue $\lambda_{1}^{2}$ for (2.1), we readily have the inequality $\|u\|_{L^{2}(\Omega)} \leq \lambda_{1}^{-2}\|\nabla u\|_{L^{2}(\Omega)}$ by Rayleigh's formula. Furthermore, for convex domains a result of Protter Pro81 demonstrates a lower bound on $\lambda_{1}^{2}$ in terms of the diameter of $\Omega$ and the radius of the largest disk contained in $\Omega$, so we could then obtain (3.11) with a constant that depends only on $\Omega$, however it would involve both spatial derivatives. Here, we will derive the constant explicitly and see that it depends on $L$.

Proof. Fix $t_{0} \geq 0$ and define

$$
F(x)=\int_{-\frac{a_{1}}{\ell} x}^{\frac{a_{2}}{\ell} x}\left|u\left(t_{0}, x, y\right)\right|^{2} d y,
$$

noting that

$$
\int_{0}^{\ell} F(x) d x=\|u\|_{L^{2}(\Omega)}^{2} .
$$

Then by Cauchy's inequality with parameter $\gamma>0$ and the fact that $\left.u\left(t_{0}, x, y\right)\right|_{\partial \Omega}=$ 0 ,

$$
\begin{aligned}
F^{\prime}(x) & =\frac{a_{2}}{\ell}\left|u\left(t_{0}, x, \frac{a_{2}}{\ell} x\right)\right|^{2}+\frac{a_{1}}{\ell}\left|u\left(t_{0}, x,-\frac{a_{1}}{\ell} x\right)\right|^{2}+2 \operatorname{Re} \int_{-\frac{a_{1}}{\ell} x}^{\frac{a_{2}}{\ell} x} u \partial_{x} \bar{u} d y \\
& \leq \gamma \int_{-\frac{a_{1}}{\ell} x}^{\frac{a_{2}}{\ell} x}|u|^{2} d y+\frac{1}{\gamma} \int_{-\frac{a_{1}}{\ell} x}^{\frac{a_{2}}{\ell} x}\left|\partial_{x} u\right|^{2} d y \\
& =\gamma F(x)+\frac{1}{\gamma} G(x)
\end{aligned}
$$

where

$$
G(x)=\int_{-\frac{a_{1}}{\ell} x}^{\frac{a_{2}}{\ell} x}\left|\partial_{x} u\left(t_{0}, x, y\right)\right|^{2} d y .
$$

Then we have

$$
F^{\prime}(x) e^{-\gamma x}-\gamma F(x) e^{-\gamma x} \leq \frac{1}{\gamma} e^{-\gamma x} G(x)
$$


and in particular,

$$
\left(F(x) e^{-\gamma x}\right)^{\prime} \leq \frac{e^{-\gamma x}}{\gamma} G(x) .
$$

By an argument following a proof of Gronwall's inequality, we see that

$$
\begin{aligned}
F(x) & \leq e^{\gamma x}\left(F(0)+\int_{0}^{x} \frac{e^{-\gamma s}}{\gamma} G(s) d s\right) \\
& =\frac{e^{\gamma x}}{\gamma} \int_{0}^{x} e^{-\gamma s} G(s) d s \\
& \leq \frac{e^{\gamma x}}{\gamma} \int_{0}^{\ell} \int_{-\frac{a_{1}}{\ell} s}^{\frac{a_{2}}{\ell} s}\left|\partial_{x} u\right|^{2} d y d s \\
& =\frac{e^{\gamma x}}{\gamma}\left\|\partial_{x} u\right\|_{L^{2}(\Omega)}^{2}
\end{aligned}
$$

and we then obtain

$$
\begin{aligned}
\|u\|_{L^{2}(\Omega)}^{2} & =\int_{0}^{\ell} F(x) d x \\
& \leq \frac{1}{\gamma^{2}}\left(e^{\gamma \ell}-1\right)\left\|\partial_{x} u\right\|_{L^{2}(\Omega)}^{2} .
\end{aligned}
$$

Choosing $\gamma=\frac{1}{\ell}$ then yields

$$
\|u\|_{L^{2}(\Omega)}^{2} \leq \ell^{2}(e-1)\left\|\partial_{x} u\right\|_{L^{2}(\Omega)}^{2} \leq L^{2}(e-1)\left\|\partial_{x} u\right\|_{L^{2}(\Omega)}^{2}
$$

since $L$ is the length of the longest side. This proves the lemma. This choice of $\gamma$ is probably not optimal, but it is sufficient for our purposes.

Combining this with 3.10 , we then have

$$
\begin{aligned}
\left|\int_{\Omega} \partial_{t} u \bar{u} d V\right| & \leq \frac{\beta}{2}\left\|\partial_{t} u\right\|_{L^{2}(\Omega)}^{2}+\frac{1}{2 \beta}\|u\|_{L^{2}(\Omega)}^{2} \\
& \leq \frac{\beta}{2}\left\|\partial_{t} u\right\|_{L^{2}(\Omega)}^{2}+\frac{L^{2}}{2 \beta}(e-1)\left\|\partial_{x} u\right\|_{L^{2}(\Omega)}^{2} \\
& \leq \frac{\beta}{2}\left\|\partial_{t} u\right\|_{L^{2}(\Omega)}^{2}+\frac{L^{2}}{2 \beta}(e-1)\|\nabla u\|_{L^{2}(\Omega)}^{2}
\end{aligned}
$$

which gives

$$
\left|\int_{\Omega} \partial_{t} u \bar{u} d V\right| \leq \frac{1}{2} L \sqrt{e-1} E(0)
$$

after choosing $\beta=L \sqrt{e-1}$.

With 3.9 and 3.22 in hand, we then obtain from (3.7) simultaneously

$$
\left|\int_{0}^{T} \int_{\partial \Omega}(X u)\left(\partial_{\nu} \bar{u}\right) d S d t\right| \leq T E(0)+2\left(2 \frac{L}{\sqrt{2}} E(0)+\frac{1}{2} L \sqrt{e-1} E(0)\right)
$$

and

$$
\left|\int_{0}^{T} \int_{\partial \Omega}(X u)\left(\partial_{\nu} \bar{u}\right) d S d t\right| \geq T E(0)-2\left(2 \frac{L}{\sqrt{2}} E(0)+\frac{1}{2} L \sqrt{e-1} E(0)\right)
$$


which we express together as the asymptotic

$$
\left|\int_{0}^{T} \int_{\partial \Omega}(X u)\left(\partial_{\nu} \bar{u}\right) d S d t\right|=T E(0)\left(1+\mathcal{O}\left(\frac{L}{T}\right)\right) .
$$

In the same fashion as in [Chr17], we now get the Neumann data on side $A$ from the term on the left hand side. Since $\left.u\right|_{\partial \Omega}=0$, the tangential derivative of $u$ along each side vanishes. On $A$, this implies $\partial_{y} u=0$. Since also $x=\ell$ and $\partial_{\nu} u=\partial_{x} u$ here, we have $X u=\ell \partial_{\nu} u$, so

$$
\begin{aligned}
\int_{0}^{T} \int_{A}(X u)\left(\partial_{\nu} \bar{u}\right) d S d t & =\int_{0}^{T} \int_{A} \ell\left(\partial_{\nu} u\right)\left(\partial_{\nu} \bar{u}\right) d S d t \\
& =\int_{0}^{T} \int_{A} \ell\left|\partial_{\nu} u\right|^{2} d S d t .
\end{aligned}
$$

On side $B$, we have $y=-\frac{a_{1}}{\ell} x$, and the unit tangent vector is $\tau=\left(\frac{\ell}{b},-\frac{a_{1}}{b}\right)$. Since the tangential derivative vanishes, we have

$$
\tau \cdot \nabla u=\frac{\ell}{b} \partial_{x} u-\frac{a_{1}}{b} \partial_{y} u=0 .
$$

The unit normal vector on $B$ is $\nu=\left(-\frac{a_{1}}{b},-\frac{\ell}{b}\right)$. Using this and (3.27), we obtain

$$
\begin{aligned}
(X u)\left(\partial_{\nu} \bar{u}\right) & =\left(x \partial_{x} u-\frac{a_{1}}{\ell} x \partial_{y} u\right)\left(-\frac{a_{1}}{b} \partial_{x} \bar{u}-\frac{\ell}{b} \partial_{y} \bar{u}\right) \\
& =x\left(-\frac{a_{1}}{b} \partial_{x} u \partial_{x} \bar{u}+\frac{a_{1}^{2}}{b \ell} \partial_{y} u \partial_{x} \bar{u}-\frac{\ell}{b} \partial_{x} u \partial_{y} \bar{u}+\frac{a_{1}}{b} \partial_{y} u \partial_{y} \bar{u}\right) \\
& =-x \frac{a_{1}}{\ell} \partial_{x} \bar{u}\left(\frac{\ell}{b} \partial_{x} u-\frac{a_{1}}{b} \partial_{y} u\right)-x \partial_{y} \bar{u}\left(\frac{\ell}{b} \partial_{x} u-\frac{a_{1}}{b} \partial_{y} u\right) \\
& =0
\end{aligned}
$$

and consequently

$$
\int_{0}^{T} \int_{B}(X u)\left(\partial_{\nu} \bar{u}\right) d S d t=0 .
$$

In a similar fashion, on side $C$ we have $y=\frac{a_{2}}{c} x$, and the unit tangent vector is $\tau=\left(\frac{\ell}{c}, \frac{a_{2}}{c}\right)$. Since the tangential derivative vanishes, we have

$$
\tau \cdot \nabla u=\frac{\ell}{c} \partial_{x} u+\frac{a_{2}}{c} \partial_{y} u=0 .
$$

The unit normal vector on $C$ is $\nu=\left(-\frac{a_{2}}{c}, \frac{\ell}{c}\right)$. Using this and 3.30, we obtain

$$
\begin{aligned}
(X u)\left(\partial_{\nu} \bar{u}\right) & =\left(x \partial_{x} u+\frac{a_{2}}{\ell} x \partial_{y} u\right)\left(-\frac{a_{2}}{c} \partial_{x} \bar{u}+\frac{\ell}{c} \partial_{y} \bar{u}\right) \\
& =x\left(-\frac{a_{2}}{c} \partial_{x} u \partial_{x} \bar{u}-\frac{a_{2}^{2}}{c \ell} \partial_{y} u \partial_{x} \bar{u}+\frac{\ell}{c} \partial_{x} u \partial_{y} \bar{u}+\frac{a_{2}}{c} \partial_{y} u \partial_{y} \bar{u}\right) \\
& =-x \frac{a_{2}}{\ell} \partial_{x} \bar{u}\left(\frac{\ell}{c} \partial_{x} u+\frac{a_{2}}{c} \partial_{y} u\right)+x \partial_{y} \bar{u}\left(\frac{\ell}{c} \partial_{x} u+\frac{a_{2}}{c} \partial_{y} u\right) \\
& =0
\end{aligned}
$$




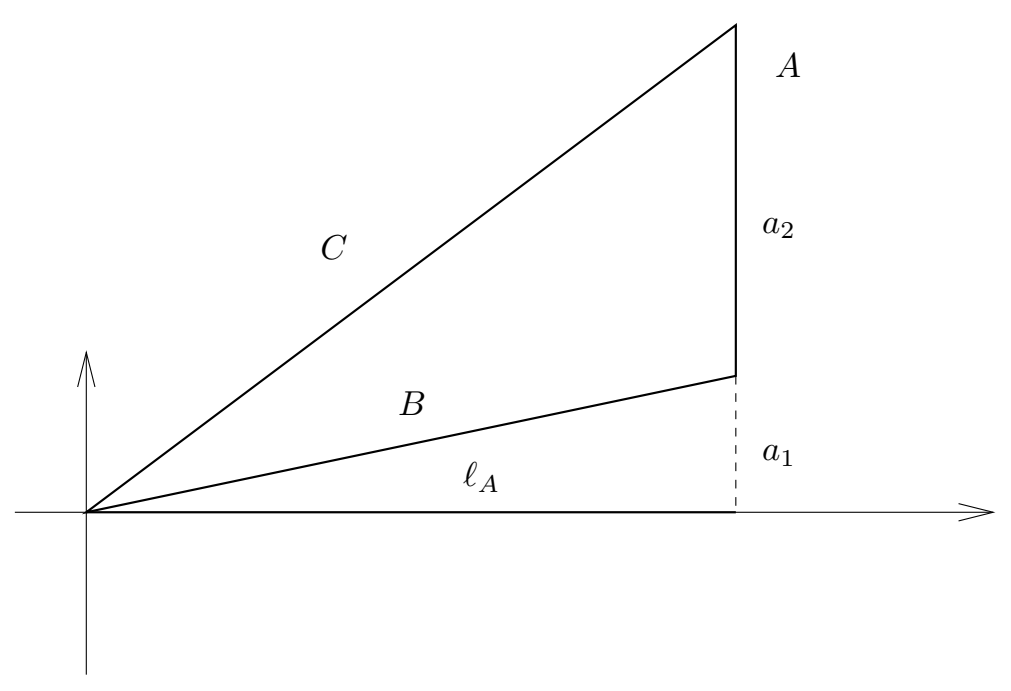

Figure 2. Setup for obtuse (and right) triangles

and consequently

$$
\int_{0}^{T} \int_{C}(X u)\left(\partial_{\nu} \bar{u}\right) d S d t=0 .
$$

Thus, from 3.29 and 3.32 we have

$$
\int_{0}^{T} \int_{\partial \Omega}(X u)\left(\partial_{\nu} \bar{u}\right) d S d t=\int_{0}^{T} \int_{A} \ell\left|\partial_{\nu} u\right|^{2} d S d t
$$

and $(3.25)$ becomes

$$
\int_{0}^{T} \int_{A} \ell\left|\partial_{\nu} u\right|^{2} d S d t=T E(0)\left(1+\mathcal{O}\left(\frac{L}{T}\right)\right)
$$

implying

$$
\int_{0}^{T} \int_{A}\left|\partial_{\nu} u\right|^{2} d S d t=\frac{T}{\ell} E(0)\left(1+\mathcal{O}\left(\frac{L}{T}\right)\right)
$$

which is the desired result.

3.2. Proof for Obtuse (or Right) Triangles. The case for an obtuse triangle plays out very similarly. Let $\Omega$ now be an obtuse (or right) triangular region in $\mathbb{R}^{2}$, as in Figure 2. Before we begin, let us observe that the proof in the acute case actually applies as long as $a_{1} \geqslant 0$ in Figure 1. That means that in Figure 2 we only need to prove the theorem for the side $A$ as the proof for sides $B$ and $C$ follow from the acute case.

Using the same vector field $X$ defined in (3.1), we have the same commutator $\left[\partial_{t}^{2}-\Delta, X\right]=-2 \Delta$ as in 3.2 . The change in domain does not affect any of the steps leading up to equation (3.7), so it still holds in this case:

$$
\int_{0}^{T} \int_{\partial \Omega}(X u)\left(\partial_{\nu} \bar{u}\right) d S d t=T E(0)+\int_{\Omega} \partial_{t} u \bar{u}+\left(\partial_{t} u\right)(X \bar{u})+\left.(X u)\left(\partial_{t} \bar{u}\right) d V\right|_{0} ^{T} .
$$


We can obtain the same bounds as in 3.9 for the last two terms, so

$$
\int_{\Omega}\left|\left(\partial_{t} u\right)(X \bar{u})\right| d V \leq \frac{L}{\sqrt{2}} E(0)
$$

holds here as well. We can also get the same bound on the first term - the argument just requires a few sign changes. In this case, the function $F$ in 3.12 must now be defined as

$$
F(x)=\int_{\frac{a_{1}}{\ell} x}^{\frac{a_{2}}{\ell} x}\left|u\left(t_{0}, x, y\right)\right|^{2} d y
$$

and we compute

$$
\begin{aligned}
F^{\prime}(x) & =\frac{a_{2}}{\ell}\left|u\left(t_{0}, x, \frac{a_{2}}{\ell} x\right)\right|^{2}-\frac{a_{1}}{\ell}\left|u\left(t_{0}, x, \frac{a_{1}}{\ell} x\right)\right|^{2}+2 \operatorname{Re} \int_{\frac{a_{1}}{\ell} x}^{\frac{a_{2}}{\ell} x} u \partial_{x} u d y \\
& \leq \gamma \int_{\frac{a_{1}}{\ell} x}^{\frac{a_{2}}{\ell} x}|u|^{2} d y+\frac{1}{\gamma} \int_{\frac{a_{1}}{\ell} x}^{\frac{a_{2}}{\ell} x}\left|\partial_{x} u\right|^{2} d y \\
& =\gamma F(x)+\frac{1}{\gamma} G(x)
\end{aligned}
$$

where the function $G$ is now

$$
G(x)=\int_{\frac{a_{1}}{\ell} x}^{\frac{a_{2}}{\ell} x}\left|\partial_{x} u\right|^{2} d y
$$

From this point the same sequence of steps applies, and we obtain

$$
\left|\int_{\Omega} \partial_{t} u \bar{u} d V\right| \leq \frac{1}{2} L \sqrt{e-1} E(0)
$$

as in 3.22. This and 3.37 imply

$$
\left|\int_{0}^{T} \int_{\partial \Omega}(X u)\left(\partial_{\nu} \bar{u}\right) d S d t\right|=T E(0)\left(1+\mathcal{O}\left(\frac{L}{T}\right)\right)
$$

mirroring 3.25 .

Finally, we obtain the Neumann data from the term on the left hand side of (3.7) via similar steps. On $A$, we again have $\partial_{y} u=0, x=\ell$, and $\partial_{\nu} u=\partial_{x} u$, so $X u=\ell \partial_{\nu} u$ and

$$
\begin{aligned}
\int_{0}^{T} \int_{A}(X u)\left(\partial_{\nu} \bar{u}\right) d S d t & =\int_{0}^{T} \int_{A} \ell\left(\partial_{\nu} u\right)\left(\partial_{\nu} \bar{u}\right) d S d t \\
& =\int_{0}^{T} \int_{A} \ell\left|\partial_{\nu} u\right|^{2} d S d t
\end{aligned}
$$

On side $B$, we have $y=\frac{a_{1}}{\ell} x$, and the unit tangent vector is $\tau=\left(\frac{\ell}{b}, \frac{a_{1}}{b}\right)$. Since the tangential derivative vanishes, we have

$$
\tau \cdot \nabla u=\frac{\ell}{b} \partial_{x} u+\frac{a_{1}}{b} \partial_{y} u=0 .
$$


The unit normal vector on $B$ is $\nu=\left(\frac{a_{1}}{b},-\frac{\ell}{b}\right)$. Using this and 3.44), we obtain

$$
\begin{aligned}
(X u)\left(\partial_{\nu} \bar{u}\right) & =\left(x \partial_{x} u+\frac{a_{1}}{\ell} x \partial_{y} u\right)\left(\frac{a_{1}}{b} \partial_{x} \bar{u}-\frac{\ell}{b} \partial_{y} \bar{u}\right) \\
& =x\left(\frac{a_{1}}{b} \partial_{x} u \partial_{x} \bar{u}+\frac{a_{1}^{2}}{b \ell} \partial_{y} u \partial_{x} \bar{u}-\frac{\ell}{b} \partial_{x} u \partial_{y} \bar{u}-\frac{a_{1}}{b} \partial_{y} u \partial_{y} \bar{u}\right) \\
& =x \frac{a_{1}}{\ell} \partial_{x} \bar{u}\left(\frac{\ell}{b} \partial_{x} u+\frac{a_{1}}{b} \partial_{y} u\right)-x \partial_{y} \bar{u}\left(\frac{\ell}{b} \partial_{x} u+\frac{a_{1}}{b} \partial_{y} u\right) \\
& =0
\end{aligned}
$$

and consequently

$$
\int_{0}^{T} \int_{B}(X u)\left(\partial_{\nu} \bar{u}\right) d S d t=0 .
$$

In a similar fashion, on side $C$ we have $y=\frac{a_{1}+a_{2}}{c} x$, and the unit tangent vector is $\tau=\left(\frac{\ell}{c}, \frac{a_{1}+a_{2}}{c}\right)$. Since the tangential derivative vanishes, we have

$$
\tau \cdot \nabla u=\frac{\ell}{c} \partial_{x} u+\frac{a_{1}+a_{2}}{c} \partial_{y} u=0 .
$$

The unit normal vector on $C$ is $\nu=\left(-\frac{a_{1}+a_{2}}{c}, \frac{\ell}{c}\right)$. Using this and 3.47), we obtain

$$
\begin{aligned}
(X u)\left(\partial_{\nu} \bar{u}\right) & \left(x \partial_{x} u+\frac{a_{1}+a_{2}}{\ell} x \partial_{y} u\right)\left(-\frac{a_{1}+a_{2}}{c} \partial_{x} \bar{u}+\frac{\ell}{c} \partial_{y} \bar{u}\right) \\
& =x\left(-\frac{a_{1}+a_{2}}{c} \partial_{x} u \partial_{x} \bar{u}-\frac{\left(a_{1}+a_{2}\right)^{2}}{c \ell} \partial_{y} u \partial_{x} \bar{u}+\frac{\ell}{c} \partial_{x} u \partial_{y} \bar{u}+\frac{a_{1}+a_{2}}{c} \partial_{y} u \partial_{y} \bar{u}\right) \\
& =-x \frac{a_{1}+a_{2}}{\ell} \partial_{x} \bar{u}\left(\frac{\ell}{c} \partial_{x} u+\frac{a_{1}+a_{2}}{c} \partial_{y} u\right)+x \partial_{y} \bar{u}\left(\frac{\ell}{c} \partial_{x} u+\frac{a_{1}+a_{2}}{c} \partial_{y} u\right) \\
& =0
\end{aligned}
$$

and consequently

$$
\int_{0}^{T} \int_{C}(X u)\left(\partial_{\nu} \bar{u}\right) d S d t=0 .
$$

Thus, from 3.46 and 3.49 we have

$$
\int_{0}^{T} \int_{\partial \Omega}(X u)\left(\partial_{\nu} \bar{u}\right) d S d t=\int_{0}^{T} \int_{A} \ell\left|\partial_{\nu} u\right|^{2} d S d t
$$

and 3.42 gives

$$
\int_{0}^{T} \int_{A} \ell\left|\partial_{\nu} u\right|^{2} d S d t=T E(0)\left(1+\mathcal{O}\left(\frac{L}{T}\right)\right),
$$

implying

$$
\int_{0}^{T} \int_{A}\left|\partial_{\nu} u\right|^{2} d S d t=\frac{T}{\ell} E(0)\left(1+\mathcal{O}\left(\frac{L}{T}\right)\right)
$$

which completes the proof of Theorem 1 . 


\section{Failure of the Result on Square Domains}

We again draw inspiration from [Chr17] to construct an example to demonstrate that our main result fails for general polygons - in particular, for squares. Let $\Omega=[0,2 \pi]^{2}$, and consider

$$
\varphi(x, y)=\frac{1}{\pi} \sin (x) \sin (n y)
$$

for some integer $n>0$. Note that $\varphi$ is a Dirichlet eigenfunction satisfying

$$
\left\{\begin{array}{l}
\left(-\Delta-\left(1+n^{2}\right)\right) \varphi=0 \\
\left.\varphi\right|_{\partial \Omega}=0
\end{array}\right.
$$

which is normalized: $\|\varphi\|_{L^{2}(\Omega)}=1$. Define now

$$
u(t, x, y)=\sin \left(t \sqrt{1+n^{2}}\right) \varphi(x, y),
$$

which satisfies

$$
\left\{\begin{array}{l}
\left(\partial_{t}^{2}-\Delta\right) u=0 \\
\left.u\right|_{\partial \Omega}=0 \\
u(0, x, y)=0, u_{t}(0, x, y)=\sqrt{1+n^{2}} \varphi(x, y)
\end{array}\right.
$$

The energy is then

$$
\begin{aligned}
E(0) & =\int_{\Omega}\left|\partial_{t} u\right|^{2}+|\nabla u|^{2} d V \\
& =\int_{\Omega}\left(1+n^{2}\right)|\varphi|^{2}+0 d V \\
& =1+n^{2} .
\end{aligned}
$$

Along the right edge $\{2 \pi\} \times[0,2 \pi]$, where $\partial_{\nu} u=\partial_{x} u$, we then have

$$
\begin{aligned}
\int_{0}^{T} \int_{0}^{2 \pi} & \left.\left|\partial_{\nu} u\right|^{2}\right|_{x=2 \pi} d y d t \\
& =\int_{0}^{T} \int_{0}^{2 \pi} \frac{1}{\pi^{2}}\left|\sin \left(\sqrt{1+n^{2}} t\right) \cos (2 \pi) \sin (n y)\right|^{2} d y d t \\
& =\frac{1}{\pi^{2}} \int_{0}^{T} \int_{0}^{2 \pi} \sin ^{2}\left(\sqrt{1+n^{2}} t\right) \sin ^{2}(n y) d y d t \\
& =\frac{1}{4 \pi^{2}} \int_{0}^{T} \int_{0}^{2 \pi}\left(1-\cos \left(2 \sqrt{1+n^{2}} t\right)\right)(1-\cos (2 n y)) d y d t \\
& =\frac{1}{4 \pi^{2}}\left(2 \pi T-\frac{2 \pi}{2 \sqrt{1+n^{2}}} \sin \left(4 \pi T \sqrt{1+n^{2}}\right)\right) \\
& =\frac{T}{2 \pi}-\frac{1}{4 \pi \sqrt{1+n^{2}}} \sin \left(4 \pi T \sqrt{1+n^{2}}\right) \\
& =\left(\frac{T}{2 \pi\left(1+n^{2}\right)}-\frac{1}{4 \pi\left(1+n^{2}\right)^{3 / 2}} \sin \left(4 \pi T \sqrt{1+n^{2}}\right)\right) E(0) .
\end{aligned}
$$

From this, we see that the analog of Theorem 1 fails to hold in this context, as an observability inequality cannot be established: there is no $T>0$ and a constant 
$C_{T}$ depending only on $\mathrm{T}$ and $\Omega$ such that

$$
\left.\int_{0}^{T} \int_{0}^{2 \pi}\left|\partial_{\nu} u\right|^{2}\right|_{x=2 \pi} d y d t \geq C_{T} E(0)
$$

for all solutions $u$, as for any $C_{T}$, a suitably large $n$ may be chosen so that 4.3 serves as a counterexample.

\section{REFERENCES}

[BC15] Nicolas Burq and Hans Christianson. Imperfect geometric control and overdamping for the damped wave equation. Comm. Math. Phys., 336(1):101-130, 2015.

[BLR92] Claude Bardos, Gilles Lebeau, and Jeffrey Rauch. Sharp sufficient conditions for the observation, control, and stabilization of waves from the boundary. SIAM J. Control Optim., 30(5):1024-1065, 1992.

[Chr07] Hans Christianson. Semiclassical non-concentration near hyperbolic orbits. J. Funct. Anal., 246(2):145-195, 2007.

[Chr10] Hans Christianson. Corrigendum to "Semiclassical non-concentration near hyperbolic orbits" [J. Funct. Anal. 246 (2) (2007) 145-195] [mr2321040]. J. Funct. Anal., 258(3):10601065, 2010.

[Chr17] Hans Christianson. Equidistribution of Neumann data mass on triangles. Proc. Amer. Math. Soc., 145(12):5247-5255, 2017.

[KN15] Nikolay Kuznetsov and Alexander Nazarov. Sharp constants in the Poincaré, Steklov and related inequalities (a survey). Mathematika, 61(2):328-344, 2015.

[Leb96] G. Lebeau. Équation des ondes amorties. In Algebraic and geometric methods in mathematical physics (Kaciveli, 1993), volume 19 of Math. Phys. Stud., pages 73-109. Kluwer Acad. Publ., Dordrecht, 1996.

[Poi90] H. Poincare. Sur les Equations aux Derivees Partielles de la Physique Mathematique. Amer. J. Math., 12(3):211-294, 1890.

[Pro81] M. H. Protter. A lower bound for the fundamental frequency of a convex region. Proc. Amer. Math. Soc., 81(1):65-70, 1981.

[Ral69] James V. Ralston. Solutions of the wave equation with localized energy. Comm. Pure Appl. Math., 22:807-823, 1969.

[RT74] Jeffrey Rauch and Michael Taylor. Exponential decay of solutions to hyperbolic equations in bounded domains. Indiana Univ. Math. J., 24:79-86, 1974.

[Ste97] V. A. Steklov. On the expansion of a given funciton into a series of harmonic funcitons. Commun. Karkov Math. Soc. Ser. 2, 6:57-124, 1897. (in Russian). 